\title{
A Fast Rendering Method for a Scene with Participating Media of Anisotropic Scattering Property
}

\author{
Yusuke Tokuyoshi and Minoru Maruyama \\ Dept. of Information Engineering, Shinshu University, 4-17-1 Wakasato, Nagano, 380-8553, Japan \\ \{ztoku, maruyama $\} @$ cs.shinshu-u.ac.jp
}

\begin{abstract}
This paper presents an efficient technique for global illumination rendering of a scene with participating media. The rendering handling participating media is performed by ray marching method, which requires sampling along each view direction. The step size of the ray marching must be taken short to generate a high quality image and thus leads to very long computational time. One possible method to improve the computational cost is to exploit importance sampling. In this paper, we propose a method to determine the step size based on the importance sampling technique. For efficient sampling, the probability density function which is "close" to the radiance distribution is required. In our method, 3D space is divided into a set of uniform grids. The radiance distribution is approximated using the grid structure. To deal with the participating media which has anisotropic scattering property, we use spherical harmonics to represent directional dependence of radiance distribution. Using this grid-based representation, fast calculation of good approximation of desirable probability density is made possible. Using this probability, high quality image can be rendered with fewer number of sampling compared to the conventional methods.
\end{abstract}

\section{Introduction}

To render a scene with natural phenomena such as beams of light through clouds, dusty air, it is necessary to simulate the effect of participating media. To render a scene with participating media, much research on volume rendering has been done. To render such a scene, volume rendering equation should be solved. The volume rendering equation usually can only be solved numerically. A typical method for solving the equation is ray marching. In the method, photon mapping is often used[2, 3].

The efficiency of computation in ray marching depends on sampling method along each ray. Adaptive sampling[2], and importance sampling based on the transmittance[1] have been proposed. In general, optimal probability distribution function for importance sampling should be proportional to the integrand. Although it is very hard (usually impossible) to obtain the exactly optimal distribution, if we can obtain good approximation rapidly, we can expect to render an image of good quality with smaller number of sampling and thus with shorter time of computation.

In this paper, we proposed a fast rendering method for a scene with participating media which can be applicable not only to isotropic media but also to media which has anisotropic scattering property. For that purpose, we propose a voxel-based radiance estimation method to obtain good probability distribution function for importance sampling. We also extend this method to deal with participating media with anisotropic scattering property. For such material, directional dependency of the radiance should be considered. In our method to obtain good approximation of inscattered radiance, spherical harmonics[4] is exploited.

To show the effectiveness of our method, we compare the image quality and computation time by our method and the conventional methods.

\section{Volume rendering methods}

In this section, we briefly review the volume rendering methods using volume photon map and ray marching.

\subsection{Light transport in participating media}

Light transported thorough a participating media is affected by emission, in-scattering, absorption, and out-scattering. Taking these four terms into account, the radiance $L$ at point $x_{k}$ in direction $\vec{\omega}$ is given as :

$$
\begin{aligned}
L\left(x_{k}, \vec{\omega}\right)= & \int_{x_{k}}^{x_{k+1}} \gamma\left(x_{k}, x\right) \alpha(x) L_{e}(x, \vec{\omega}) d x \\
& +\int_{x_{k}}^{x_{k+1}} \gamma\left(x_{k}, x\right) \sigma(x) L_{i}(x, \vec{\omega}) d x \\
& +\gamma\left(x_{k}, x_{k+1}\right) L\left(x_{k+1}, \vec{\omega}\right)
\end{aligned}
$$


where $L_{e}$ is the emitted radiance, $L_{i}$ is the in-scattered radiance, $\alpha$ is absorption coefficient, $\sigma$ is scattering coefficient, and $\gamma\left(x_{k}, x\right)$ is transmittance along the line segment from $x_{k}$ to $x$. This equation is called as the volume rendering equation. This equation must be solved in order to render a scene with participating media.

The transmittance $\gamma\left(x_{k}, x\right)$ is given by :

$$
\gamma\left(x_{k}, x\right)=e^{-\int_{x_{k}}^{x} \kappa(\tau) d \tau}
$$

where the extinction coefficient $\kappa$ is defined as $\kappa=\alpha+\sigma$.

The in-scattered radiance $L_{i}$ is

$$
L_{i}(x, \vec{\omega})=\int_{\Omega} p\left(\vec{\omega}^{\prime} \cdot \vec{\omega}\right) L\left(x, \vec{\omega}^{\prime}\right) d \vec{\omega}^{\prime}
$$

Where $p\left(\vec{\omega}^{\prime} \cdot \vec{\omega}\right)$ is the phase function of the medium. Jansen proposed an efficient method to compute inscattered radiance using volume photon map[2, 3]. The volume photon map stores photons which are scattered in participating media. We can estimate the $L_{i}$ using the volume photon map as :

$$
L_{i}(x, \vec{\omega}) \approx \frac{1}{\kappa(x)} \sum_{p=0}^{N} p\left(\vec{\omega}_{p} \cdot \vec{\omega}\right) \frac{\Delta \Phi\left(x, \vec{\omega}_{p}\right)}{d V}
$$

Where $d V$ is small volume, $\Delta \Phi$ is flux of photon.

\subsection{Ray marching}

It is difficult to solve the rendering equation analytically. The volume rendering equation can be solved by using numerical integration. To evaluate the integral, ray marching method is often used. In ray marching, the ray is divided into small segments. Within each segment, we assume incoming light is constant and the properties of media is not changed. With the local simplification, we can approximate the radiance $L\left(x_{k}, \vec{\omega}\right)$ as :

$$
\begin{aligned}
L\left(x_{k}, \vec{\omega}\right)= & \Delta x \alpha\left(x_{k}\right) L_{e}\left(x_{k}, \vec{\omega}\right) \\
& +\Delta x \sigma\left(x_{k}\right) L_{i}\left(x_{k}, \vec{\omega}\right) \\
& +e^{-\Delta x \kappa\left(x_{k}\right)} L\left(x_{k+1}, \vec{\omega}\right)
\end{aligned}
$$

where $\Delta x=\left|x_{k+1}-x_{k}\right|$ is segment size. The segment size of the ray marching must be taken short to generate a high quality image and thus usually leads to very long computational time.

\subsection{Optimization methods}

The typical ray marcher described in Section 2.2 is usually based on a uniform step size through the medium. For non-homogeneous media and media with local variations in the lighting, it is preferable to use adaptive ray marching. In adaptive ray marching method, segment size is determined adaptively to capture local changes efficiently. The simplest method for doing this is a recursive ray marcher, where the midpoint of the segment is sampled recursively until the length of the segment is below a certain threshold, or until the discrepancy between the endpoints of the segment is sufficiently small.

Another possible method is to use importance sampling technique based on transmittance. Importance sampling is a variance reduction technique in Monte-Carlo simulation. If the media is homogeneous between $x_{k}$ and $x_{k+1}$, volume rendering equation using importance sampling is given as follows :

$$
\begin{aligned}
L\left(x_{k}, \vec{\omega}\right)= & S\left(x_{k}, \Delta x\right) \alpha\left(x_{k}\right) L_{e}\left(x_{k}, \vec{\omega}\right) \\
& +\Delta L\left(x_{k}, \vec{\omega}, \Delta x\right) \\
& +e^{-\Delta x \kappa\left(x_{k}\right)} L\left(x_{k+1}, \vec{\omega}\right)
\end{aligned}
$$

where $S$ is derived from the first term of (1) by integrating $\int_{x_{k}}^{x_{k+1}} \gamma\left(x_{k}, x\right) d x$.

$$
S(x, \Delta x)=\frac{1-e^{-\Delta x \kappa(x)}}{\kappa(x)}
$$

$\Delta L(x, \vec{\omega}, \Delta x)$ represents the approximation of the second term of (1). This is given by using importance sampling method as follows :

$$
\Delta L(x, \vec{\omega}, \Delta x)=\frac{\sigma(x)}{M} \sum_{j=0}^{M} \frac{e^{-t_{j} \kappa(x)} L_{i}\left(x+t_{j} \vec{\omega}, \vec{\omega}\right)}{P\left(t_{j}\right)}
$$

where $P$ is pdf (probability density function) for importance sampling and $t$ is distance along the ray. $t_{j}$ represents the sampling point according to the pdf $P$. In general, to reduce the variance in Monte-Carlo simulation, it is desirable to use the probability density function $P$ whose shape is close to the integrand (i.e. in the case of volume rendering equation : $\left.e^{-t_{j} \kappa\left(x_{k}\right)} L_{i}\left(x_{k}+t_{j} \vec{\omega}, \vec{\omega}\right)\right)$. For simplicity, the following pdf is often used :

$$
\begin{gathered}
P(t)=\frac{e^{-t \kappa(x)}}{S(x, \Delta x)} \\
t=-\frac{\log \xi}{\kappa}
\end{gathered}
$$

where $\xi$ is uniformly distributed random number on $\left[0, e^{-\Delta x \kappa}\right]$.

Using (9), $\Delta L(x, \vec{\omega}, \Delta x)$ is given by : 


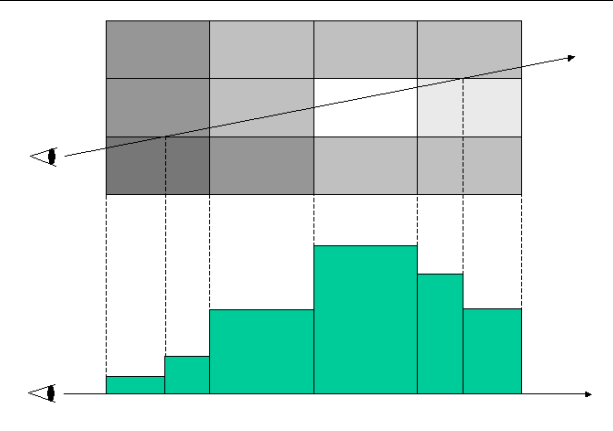

Figure 1. Density estimation from the voxels in the grid

$$
\Delta L(x, \vec{\omega}, \Delta x)=\frac{S(x, \Delta x) \sigma(x)}{M} \sum_{j=0}^{M} L_{i}\left(x+t_{j} \vec{\omega}, \vec{\omega}\right)
$$

\subsection{Further improvement by pdf estimation}

We have briefly reviewed both adaptive sampling and simplified importance sampling. Efficiency of adaptive sampling depends on the initial segment size. When the strong radiance is concentrated on the small region, the initial segment size should be smaller than the size of the effect. Thus, without specific prior knowledge on the scene, we have to use very small initial segment. As the result, this gives rise to long computational time.

As for the importance sampling, ideally the probability distribution function should be proportional to the integrand of the volume rendering equation. Assuming the homogeneity of media along the segment, the ideal probability distribution function $P_{x_{k}, \vec{\omega}}(t)$ should be :

$$
P_{x_{k}, \vec{\omega}}(t) \propto e^{-t \kappa\left(x_{k}\right)} L_{i}\left(x_{k}+t \vec{\omega}, \vec{\omega}\right)
$$

Usually, for simplicity, $L_{i}\left(x_{k}+t \vec{\omega}, \vec{\omega}\right)$ is treated as constant, as shown above. However, this is a very rough approximation. If we can obtain near-optimal probability distribution function rapidly, due to the variance reduction effect, we can expect to render an image of good quality with smaller number of sampling, in other words shorter time of computation. In the following sections, we explore the importance sampling method based on the estimation of the in-scattered radiance.

\section{Importance sampling based on the estima- tion of in-scattered radiance}

To obtain more accurate probability distribution function for importance sampling, in-scattered radiance $L_{i}$ should be considered. However, usually, $L_{i}$ is a very complicated function and it is time consuming to calculate accurate estimation of the function value.

Our goal is to obtain fast volume rendering algorithm based on the importance sampling along the ray. If we can obtain good probability distribution function rapidly, due to the variance reduction effect, we can expect to render an image of good quality with smaller number of sampling and thus can reduce the computational cost. For that purpose, fast and efficient method to approximate in-scattered radiance is required. In the following subsections, we propose an efficient method to approximate in-scattered radiance $L_{i}$, which is applicable even for media with anisotropic scattering property.

\subsection{Approximation of radiance using uniform grid}

In our work, we try to derive probability distribution function for importance sampling by considering in-scattered radiance $L_{i}$. One obvious method is to approximate $L_{i}(x, \vec{\omega})$ by querying the volume photon map at each point $x$. However, by this method, the number of access to the photon map would get prohibitive. This implies this simple method is not appropriate for fast rendering.

To reduce the computational cost, we use uniform grid structure and represent 3D space by a set of voxels. At each voxel center $c$, we estimate $L_{i}(c, \vec{\omega})$ by the above simple method using volume photon map. The in-scattered radiance at the other point $x$ within the voxel is approximated by the representative value $L_{i}(c, \vec{\omega})$ instead of querying photon map. This reduces computational cost significantly.

From the voxels which are intersected with a ray, we can obtain the histogram of approximated radiance (see Figure.1). From the histogram, the probability distribution function for importance sampling is calculated based on (12).

To make the histogram, we have to rapidly find the voxels which are intersected with each ray. Since we use uniform grid, this is made possible by using 3DDDA (3D Digital Difference Analyzer) algorithm.

There are two tasks to be done when we create a grid. The first is, given the axis aligned bounding box, to choose the number of subdivisions. The second is to save the estimated radiance value from photon map to the grid.

It has been argued that the grid cells should be roughly cubes and number of grid cells should be the same order of 

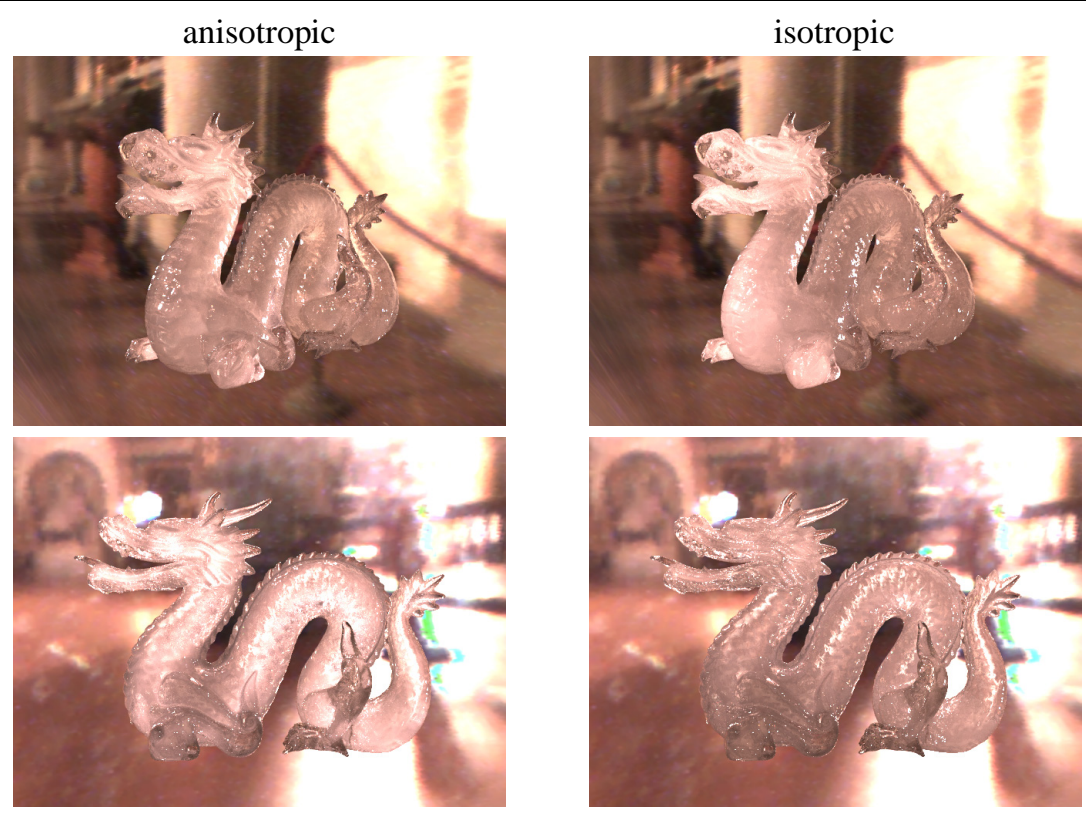

Figure 2. rendering examples : participating media with anisotropic scattering property and isotropic scattering property

magnitude as the number of elements (i.e. photons)[6]. This can be accomplished in the follow manner.

$$
\begin{gathered}
s=\left(\frac{a N}{k w_{x} w_{y} w_{z}}\right)^{\frac{1}{3}} \\
n_{x}=w_{x} s, n_{y}=w_{y} s, n_{z}=w_{z} s
\end{gathered}
$$

where $w_{x}, w_{y}$ and $w_{z}$ are the lengths of the bounding box sides. $n_{x}, n_{y}$ and $n_{z}$ are the number of subdivision used to create $n_{x} n_{y} n_{z}$ grid. $N$ is the number of photons in the photon map. $k$ is the number of photons used to estimate radiance for each point. $a$ is parameter of grid quality. The order of computational cost of radiance estimation is given as $O(k+\log N)$. The total number of cells are $N_{c}=n_{x} n_{y} n_{z}$. Therefore, the total computational cost of grid generation is given as $O\left(N_{c} k+N_{c} \log N\right)$.

\subsection{Approximation using spherical harmonics}

If the participating media has the isotropic phase function, $L_{i}(x, \vec{\omega})$ does not depend on $\vec{\omega}$. Therefore, in this case, we don't have to care about the directional dependency of the in-scattered radiance $L_{i}(x, \vec{\omega})$. It is sufficient to store scalar value for each voxel.

However, in the real world, there are many kinds of materials which have anisotropic scattering properties. To render a scene with participating media which has anisotropic scattering property, directional dependency should be taken into consideration. As the result, the probability distribution function for the importance sampling should also be decided reflecting the anisotropic scattering property of the participating media. In Figure 2, we show example images of scenes with isotropic media and anisotropic media.

To represent the directional dependency of the inscattered radiance $L_{i}(x, \vec{\omega})$ concisely, we approximate the radiance at voxel centers by using spherical harmonics[4] as follows :

$$
L_{i}(x, \vec{\omega})=\sum_{l=0}^{\infty} \sum_{m=-l}^{l} f_{x, l, m} Y_{l, m}(\vec{\omega})
$$

where $Y_{l, m}$ is spherical harmonic basis. $f_{l, m}$ is an associated expansion coefficient. $L_{i}$ is approximated by finite number of coefficients and basis functions as:

$$
L_{i}(x, \vec{\omega}) \approx \sum_{l=0}^{K} \sum_{m=-l}^{l} f_{x, l, m} Y_{l, m}(\vec{\omega})
$$

The coefficients $f_{l, m}$ are stored in each voxel. The total number of the coefficients are $(K+1)^{2}$. To approximate the radiance, as shown in the above equation, $Y_{l, m}(\vec{\omega})$ should also be calculated. For each ray, this computation is performed only once for each pair $(l, m)$. This is advantageous for fast computation of radiance value along each ray.

The expansion coefficients are obtained as follows: 


$$
f_{x, l, m}=\int_{\Omega} L_{i}(x, \vec{\omega}) Y_{l, m}^{*}(\vec{\omega}) d \vec{\omega}
$$

where $Y^{*}$ is complex conjugate of $Y$. These coefficients are calculated efficiently by expanding the phase function into Legendre polynomials (see Appendix).

\subsection{Sampling method}

In our work, the probability distribution function for importance sampling is derived by using estimation of inscattered radiance $L_{i}$ and transmittance which is shown in (2). The radiance along the ray is approximated using histogram representation described in the previous subsection. The histogram is generated by querying coefficients of spherical harmonics stored in the voxels which are intersected with the ray. The probability distribution function $P(t)$ is given by :

$$
\begin{aligned}
P_{x, \vec{\omega}}(t) & =\frac{h_{x, \vec{\omega}}(t) e^{-t \kappa(x)}}{\int_{0}^{\Delta x} h_{x, \vec{\omega}}\left(t^{\prime}\right) e^{-t^{\prime} \kappa(x)} d t^{\prime}} \\
& =\frac{h_{x, \vec{\omega}}(t) e^{-t \kappa(x)}}{S_{h}(x, \vec{\omega})}
\end{aligned}
$$

where $t$ is the distance parameter along the ray, $S_{h}$ is given by the following equation.

$$
S_{h}(x, \vec{\omega})=\sum_{i=0}^{n} h_{x, \vec{\omega}, i} S\left(x, w_{x, \vec{\omega}, i}\right)
$$

( $S$ is given by (7))

$\Delta L(x, \vec{\omega}, \Delta x)$ based on the importance sampling is given by :

$$
\Delta L(x, \vec{\omega}, \Delta x)=\frac{\sigma(x) S_{h}(x, \vec{\omega})}{M} \sum_{j=0}^{M} \frac{L_{i}\left(x+t_{j} \vec{\omega}, \vec{\omega}\right)}{h_{x, \vec{\omega}}\left(t_{j}\right)}
$$

where $h$ is an estimation of in-scattered radiance $L_{i}$ by using the histogram. We can expect the following relation holds :

$$
L_{i}\left(x_{k}+t_{j} \vec{\omega}, \vec{\omega}\right) \approx h_{x_{k}, \vec{\omega}}\left(t_{j}\right)
$$

The probability distribution for sampling is given by (16). The total number of samples is determined according to (17). With this procedure, more samples are used in the important parts within an image. This is important to render an image in which the image quality is almost homogeneous.

In our method, we approximate the probability distribution function for importance sampling by using spherical

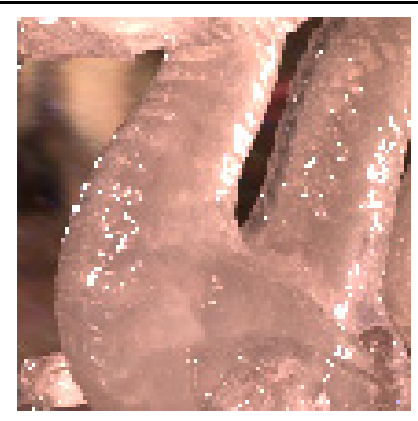

reference image

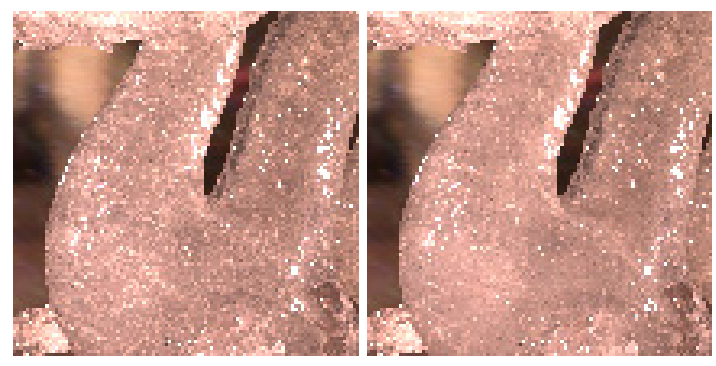

(a) Dragon (same as top left of Fig.2)
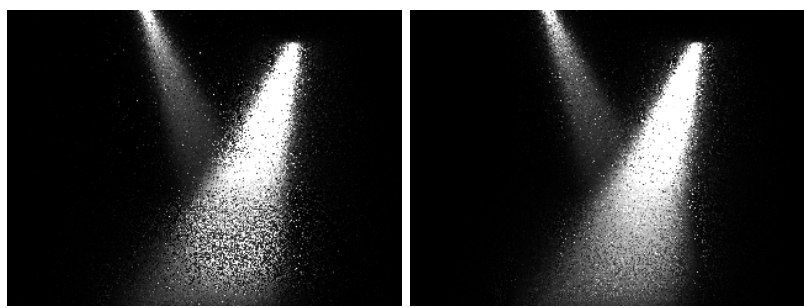

(b) Two spotlights in anisotropic scattering medium

Figure 3. Comparison of images rendered with PDFs derived using scalar representation (one coefficient for each cell) and spherical harmonics (16 coefficients for each cell): Left image is rendered using scalar representation. Right image is rendered using spherical harmonics.

harmonics to reflect the directional dependency of the radiance. If the participating media have the isotropic phase function, we may use the representation in which each grid cell has a scalar value.

To compare these two types of the representation, we have rendered a couple of scenes based on the PDFs derived using scalar representation (one coefficient for each cell) and the spherical harmonics (16 coefficients for each cell). We show the results in Figure 4. These images indicate the approximation method based on the spherical harmonics is preferable for anisotropic participating media. 


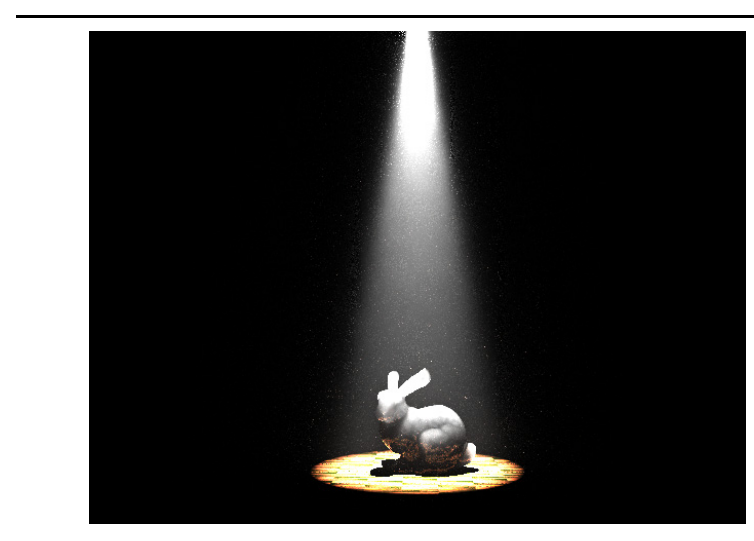

Figure 4. The example of strong radiance on small region: The image is rendered using our method in 3 minutes. To Approximate in-scattered radiance distribution, kd-tree is used.

\subsection{Hierarchical Data Structure}

We approximated in-scattered radiance using uniform grid in section 3.1. To further improve the approximation quality, we can use hierarchical data structures such as kdtree or octree. Hierarchical data structures are efficient in a scene that has sharp illumination edges. Dividing volume with big local changes in in-scattered radiance into many cells, approximation quality can be further improved. Especially, this adaptive cell division technique is effective for a scene that has strong radiance on small regions (Figure 4).

In principle, the cell with big local change in radiance should be divided recursively. However, in our method, subdivision of cells are carried out based on density of the volume photon map. Although the region with big local change does not necessarily correspond to the bright region in a scene, as the experiments show our method works well in practice.

\section{Experimental Results}

We have rendered a couple of scenes by using the proposed method and for comparison by using the conventional methods. We used two conventional methods. They are :

(i) uniform sampling described in (5) with random offset to eliminate the aliasing problems.

(ii) importance sampling based on transmittance described in (11).

In Figure 5 we compare the image quality by theses methods.

In experiment \#1, we used a point light source. In experiment \#2, planar light source which emits directional light was used. In both experiment \# 1 and experiment \#2, each object has surface which obeys Fresnel equations. In the both experiments, outside the object, there exists participating media which has isotropic scattering property whose extinction coefficient is 1.0. In the experiment \#1, inside the object, there exists anisotropic media which gives forward scattering and whose extinction coefficient is 50 . In the experiment \# 2 , inside the object, there also exits forward scattering media whose extinction coefficient is 200 .

In the both of the experiments, the size of the rendered images was $640 \times 480$ pixels. In the experiments, 100000 photons were used. To approximate directional dependency of the in-scattered radiance, 16 coefficients of spherical harmonics were used. The images were rendered by a PC with 2.8GHz Pentium 4 CPU.

In Figure 5, we show the resultant images. Top row shows the results by uniform sampling along the ray. In the middle row, we show the results by importance sampling with the pdf given by (8) (pdf which is simply decided assuming the constant radiance). In the bottom row, results by the proposed method are shown.

In Figure 5, each column shows the images rendered with same computation time for comparison. Time required for generating grid structure was $7.0 \mathrm{sec}$ in experiment \#1, $8.4 \mathrm{sec}$ in experiment \# 2 .

As the images indicate, the proposed method outperformed the other methods. Especially, the image quality by our method was almost homogeneous. On the other hand, by the other methods, there exist differences in image quality within each image. Some parts were much more noisy compared to the other parts of a image. The homogeneity in the resultant image and fast computation are the advantageous points of our proposed method.

Method (i) and method (ii) depend on the strong assumption on the distribution of in-scattered radiance. Sometimes, this assumption is too strong. For example, if scenes have strong radiance on small regions (e.g. volume caustics, direct lights), to render a noiseless image with these method, a lot of sample points are required. On the other hand, in our method, sampling is carried out by considering both the transmittance and the approximation of radiance distribution. As the experiments showed, even if the strong radiance exists on small regions in a scene, our method can render high quality image with fewer sample points.

\section{Conclusion}

In this paper, we proposed a fast rendering method for a scene with participating media. Our method is applicable not only to isotropic media but also to media which has anisotropic scattering property. Our method is based on ray marching algorithm using the photon map $[2,3]$. Importance sampling is used to decide the segment size along the ray 
for ray marching. In general, to reduce variance in MonteCarlo integrand, the optimal probability distribution function should be proportional to the integrand. Although it is very hard (usually impossible) to get the optimal distribution, if we can obtain good approximation rapidly, we can expect to render an image of good quality with smaller number of sampling. This can lead to significant reduction of rendering time.

For that purpose, we have proposed a voxel-based radiance estimation method to obtain probability distribution function for importance sampling. We have also extended this method to deal with participating media with anisotropic scattering property. For such material, directional dependency of the radiance should be considered. In our method to obtain good approximation of in-scattered radiance, spherical harmonics is exploited.

The rendering experiments showed our method can outperform conventional rendering methods with respect to rendering speed and the image quality.

\section{References}

[1] P. Dutre, P. Bekaert, and K. Bala: Advanced global illumination, A K Peters.(2003)

[2] H. W. Jensen and P. H. Christen: Efficient simulation of light transport in scenes with participating media using photon maps, Proc SIGGRAPH '98, pp.311-320,(1998)

[3] H. W. Jensen: Realistic image synthesis using photon mapping, A K Peters.(2001)

[4] J. T. Kajiya and B. P. Von Herzen: Ray tracing volume densities, Proc SIGGRAPH '84, pp.165-174(1984)

[5] J. Kautz, P. Sloan, J. Snyder: Fast, arbitrary BRDF shading for low-frequency lighting using spherical harmonics, Eurographics Rendering Workshop '2002, pp.291-296(2002)

[6] P. Shirley: Realistic ray tracing, A K Peters. pp.71-76(2000)

[7] P. Sloan, J. Kautz, J. Snyder: Precomputed radiance transfer for real-time rendering for dynamic, low-frequency lighting environments, ACM Transactions on Graphics 21, 3, pp.527536(2002)

[8] The Stanford 3D Scanning Repository. http://graphics.stanford.edu/data/3Dscanrep/

\section{A. Derivation of coefficients of spherical har- monics}

We expand radiance $L_{i}(\mathbf{x}, \vec{\omega})$ into spherical harmonics. The coefficients $f_{l, m}$ is given by:

$$
f_{l, m}=\int_{\Omega} L_{i}(\vec{\omega}) Y_{l, m}^{*}(\vec{\omega}) d \vec{\omega}
$$

From the equation (4),

$$
f_{l, m}=\int_{\Omega} \sum_{p=0}^{N} \frac{p\left(\vec{\omega}_{p} \cdot \vec{\omega}\right) \Delta \Phi\left(\vec{\omega}_{p}\right)}{\kappa d V} Y_{l, m}^{*}(\vec{\omega}) d \vec{\omega}
$$

The phase function can be expanded with Legendre polynomials as :

$$
p\left(\vec{\omega}_{p} \cdot \vec{\omega}\right)=\sum_{k=0}^{\infty} w_{k} P_{k}\left(\vec{\omega}_{p} \cdot \vec{\omega}\right)
$$

where $P_{k}\left(\vec{\omega}_{p} \cdot \vec{\omega}\right)$ is Legendre polynomial. Coefficient $w_{k}$ is given by :

$$
w_{k}=\frac{2 k+1}{2} \int_{-1}^{1} p(u) P_{k}(u) d u
$$

Since the Legendre polynomial can be represented with spherical harmonics [4] as :

$$
P_{k}\left(\vec{\omega}_{p} \cdot \vec{\omega}\right)=\frac{4 \pi}{2 k+1} \sum_{i=-k}^{k} Y_{k, i}(\vec{\omega}) Y_{k, i}^{*}\left(\vec{\omega}_{p}\right)
$$

the phase function can be expressed as follows :

$$
p\left(\vec{\omega}_{p} \cdot \vec{\omega}\right)=\sum_{k=0}^{\infty} w_{k}^{\prime} \sum_{i=-k}^{k} Y_{k, i}(\vec{\omega}) Y_{k, i}^{*}\left(\vec{\omega}_{p}\right)
$$

where $w_{k}^{\prime}$ is obtained by :

$$
w_{k}^{\prime}=2 \pi \int_{-1}^{1} p(u) P_{k}(u) d u
$$

By substituting (21) into (25), the coefficients $f_{l, m}$ is obtained as follows.

$$
f_{l, m}=\frac{w_{l}^{\prime}}{\kappa} \sum_{p=0}^{N} \frac{\Delta \Phi\left(\vec{\omega}_{p}\right)}{d V} Y_{l, m}^{*}\left(\vec{\omega}_{p}\right)
$$




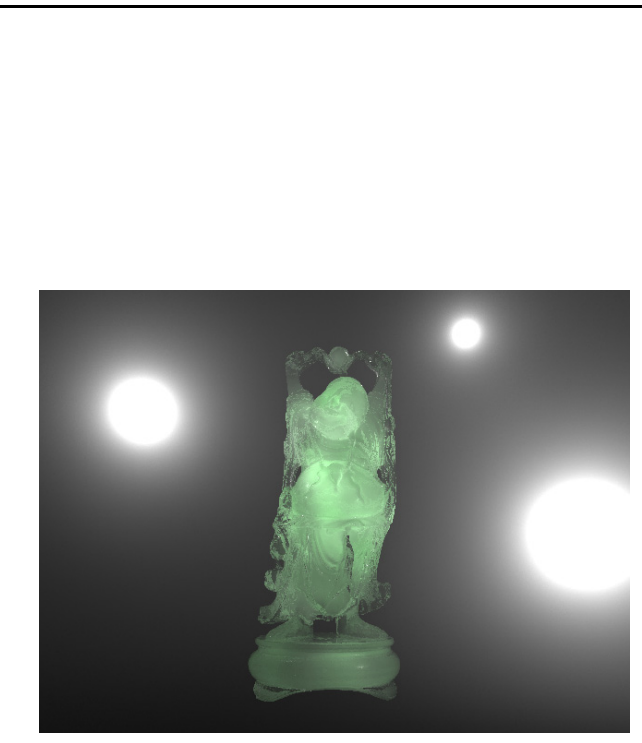

reference image

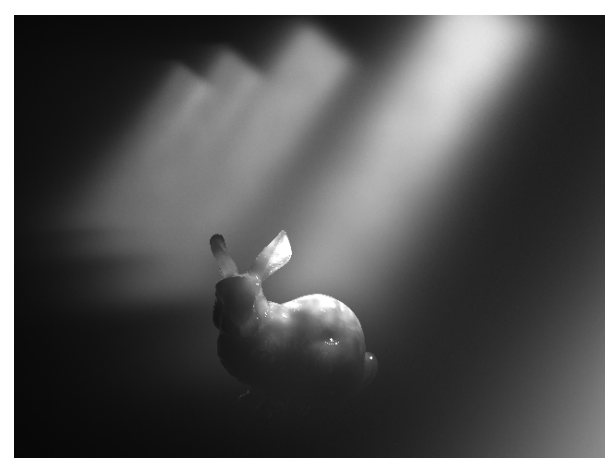

reference image

\section{Experiment \#1}

$8 \min$

(a)

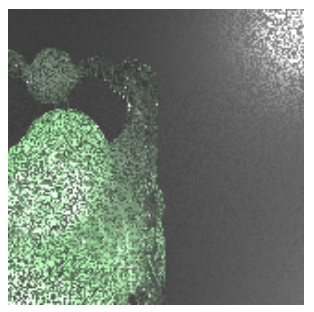

(b)

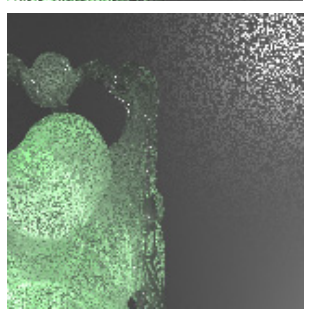

(c)

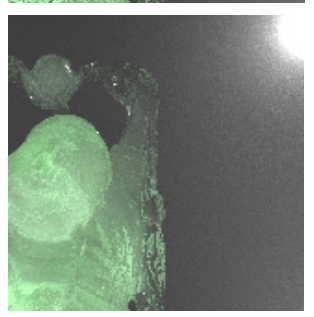

Experiment \#2

$4 \mathrm{~min} 45 \mathrm{sec}$

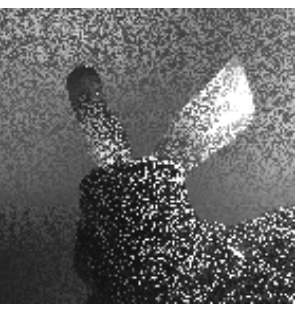

(a)

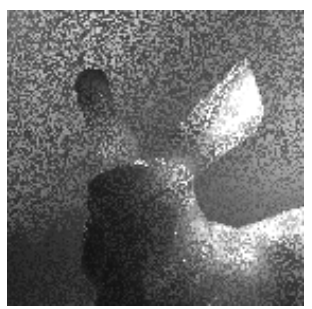

(b)

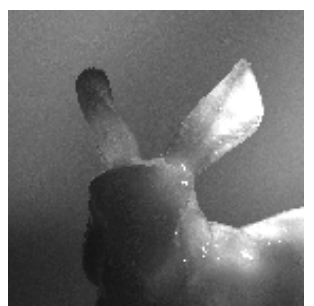

$24 \mathrm{~min}$
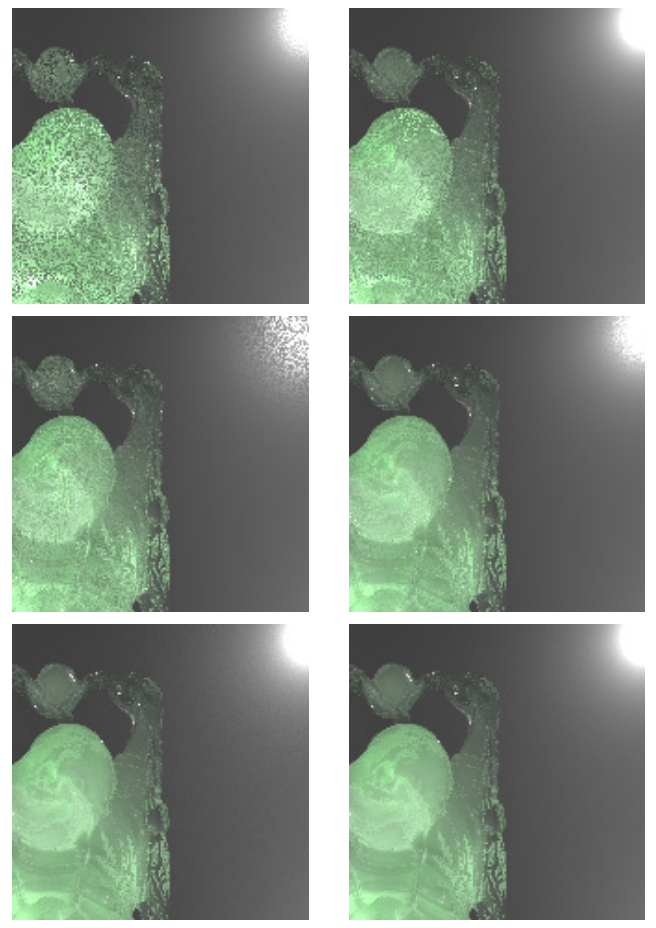

$45 \mathrm{~min}$
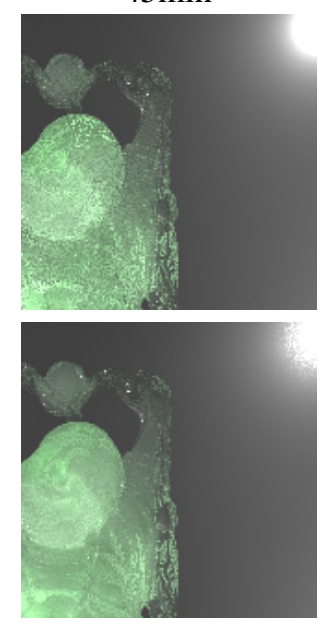
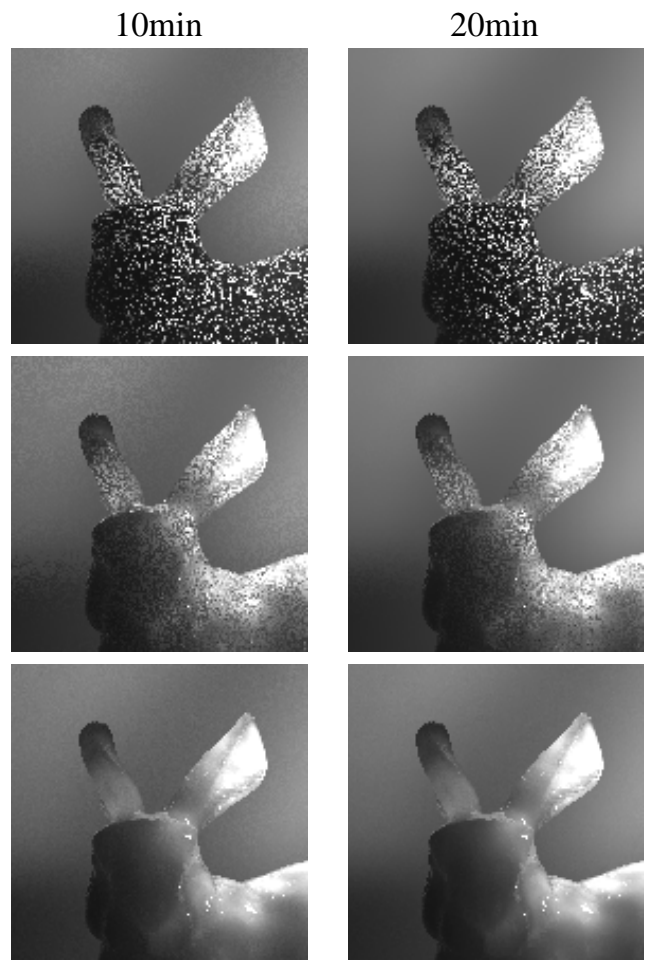

Figure 5. comparison of image quality : (a) uniform sampling. (b) importance sampling based on transmittance. (c) importance sampling based on our method. 